University of Wollongong

Research Online

Faculty of Engineering and Information

Faculty of Engineering and Information

Sciences - Papers: Part B

Sciences

2017

\title{
Assisting Open Education Resource Providers and Instructors to Deal With Cold Start Problem in Adaptive Micro Learning: a Service Oriented Solution
}

Geng Sun

University of Wollongong, gs147@uowmail.edu.au

Tingru Cui

University of Wollongong, tingru@uow.edu.au

Dongming $\mathrm{Xu}$

University of Queensland, d.xu@business.uq.edu.au

Huaming Chen

University of Wollongong, hc007@uowmail.edu.au

Shiping Chen

CSIRO ICT Centre, shiping.chen@csiro.au

See next page for additional authors

Follow this and additional works at: https://ro.uow.edu.au/eispapers1

Part of the Engineering Commons, and the Science and Technology Studies Commons

Research Online is the open access institutional repository for the University of Wollongong. For further information contact the UOW Library: research-pubs@uow.edu.au 


\section{Assisting Open Education Resource Providers and Instructors to Deal With Cold Start Problem in Adaptive Micro Learning: a Service Oriented Solution}

\section{Keywords}

start, problem, adaptive, micro, learning:, open, solution, education, resource, assisting, providers, instructors, deal, cold, oriented, service

Disciplines

Engineering | Science and Technology Studies

\section{Publication Details}

Sun, G., Cui, T., Xu, D., Chen, H., Chen, S. \& Shen, J. (2017). Assisting Open Education Resource Providers and Instructors to Deal With Cold Start Problem in Adaptive Micro Learning: a Service Oriented Solution. IEEE SCC 2017: IEEE International Conference on Services Computing (pp. 196-203). United States: IEEE.

\section{Authors}

Geng Sun, Tingru Cui, Dongming Xu, Huaming Chen, Shiping Chen, and Jun Shen 


\title{
Assisting Open Education Resource Providers and Instructors to Deal With Cold Start Problem in Adaptive Micro Learning: a Service Oriented Solution
}

\author{
Geng Sun ${ }^{1}$, Tingru Cui ${ }^{1}$, Dongming $\mathrm{Xu}^{2}$, Huaming Chen ${ }^{1}$, Shiping Chen ${ }^{3}$, Jun Shen ${ }^{1}$ \\ 1, School of Computing and Information Technology, University of Wollongong, Wollongong, Australia \\ 2, UQ Business School, The University of Queensland, Brisbane, Australia \\ 3, CSIRO Data61, Australia \\ Email: gs147@uowmail.edu.au, tingru@uow.edu.au,D.Xu@business.uq.edu.au,hc007@uowmail.edu.au, \\ Shiping.Chen@data61.csiro.au, jshen@uow.edu.au
}

\begin{abstract}
Various prior studies have leveraged cloud computing and big data techniques to promote adaptive micro open learning. However, this novel way of open education resource (OER) delivery and access suffers from the cold start problem of learner information. In this paper, we introduce a service oriented solution to assist OER providers and instructors to deal with the sparsity of data in OER recommendation using an ontological approach. Learners' features are predicted by spreading activation and demographic similarity based inference. An evolutionary algorithm is provided to realize the OER recommendation in terms of heuristic rules.
\end{abstract}

Keywords-Cold Start, Software as a Service, Semantic Inference, Open Education Resource, Micro Learning

\section{INTRODUCTION}

In the information age, the development and dissemination of learning resources are booming in a much higher speed and wider range than their traditional shape. Along with various leading universities opening up access to their courses, open education resources (OERs) are becoming available exponentially. The rise of OERs gains large popularity in the entire higher and adult education sector, and these novel learning paradigms have attracted many researchers' attention, from educational, social, and computational views [1]. According to the latest statistics, millions of people have attended the virtual classrooms of online open learning to access OERs, which are produced and updated on a daily basis. Using OER, as claimed, anyone from anywhere is given an equal opportunity to take courses and have access to educational resources that they otherwise could not afford. This leads to the emerging trend - open learning [2].

People have shown increasing interests in getting access to online learning resources and participating in online learning activities via electronic, especially mobile devices [3]. On the other hand, the increasingly frequent use of mobile devices and fast-approaching life paces bring in the trend of micro learning.

OER providers and instructors have tried to promote their courses and affiliated educational products at full stretch. They have leveraged mobile learning (m-learning) for learners to easily participate in learning activities regardless of restrictions in time and location. Web service and cloud based approaches are also commonly seen in mainstream OER products with respect to the purpose of enhancing the system operation performance and user experience.

However, many educational professionals are still evaluating the cons and pros of the OER to foresee if it can act as a regular, or barely complementary, pedagogical approach for m-learning. In addition, because of the inventiveness of the new education trend, its popularity has been limited by the lack of personalized services so that current OER delivery often fails to meet comparatively diverse demands from both OER providers and learners. In other words, it is anticipated to have OER delivered in a personalized learning environment (PLE) rather than just a traditional virtual learning environment (VLE). Moreover, these OERs are suggested to be consumed in a micro learning mode which conforms to the custom of the modern e-society.

In our pilot work, we proposed a software as a service, Micro learning as a Service (MLaaS) to support adaptive micro learning through OERs [4]. The working principle of MLaaS will be briefly introduced in the Section III, which functions in provide intelligent OER collection and recommendation through computing. This trial was influenced by the shortage of learner information, which leads to the difficulty in making a computational decision of micro OER adaptations. This motivated us to come up with a cloud based solution, which will be introduced as the main contribution of this paper. This solution aims to build a knowledge base to evidently support the first decisionmaking process of micro adaptation, including learner feature prediction and demographic similarity based inference. Its technical details are mainly located in the Section V. Please also note that we will use the terms micro learning through OER and micro open learning interchangeably in the following sections.

\section{BACKGROUND}

\section{A. OER and OER as a Service}

Open learning, which aims to integrate e-learning and open courses with other learning modes, is vigorously pursued by many education providers. It has been adopted by many regional universities or universities with multiple 
campus operations. It is quite different from on-campus, e/m-learning mode. OERs are "digital learning resources offered online freely and openly to teachers, educators, students, and independent learners in order to be used, shared, combined, adapted, and expanded in teaching, learning and research" [2]. Open learning has the advantage of both informal learning and formal learning. Learners enjoy high flexibilities of online open learning because there is no strict time constraint for joining and quitting. Learners engaged in open learning are from different age groups and culture backgrounds with a wide range of geographic distribution.

There are still some barriers for course instructors to migrant their previous teaching strategies to this new blended learning environment. Thanks to the advantages of services oriented and mobile cloud-based learning and forthcoming mobile apps, delivering OER in a serviceoriented mode is of substantial potential to be employed by OER providers. For example, a trial case, OER as a Service, is not only believed to have potential in OER distribution, but also eases the process of service deployment and delivery [5][6]. Service-oriented OER provision encloses the content service, technical service and customer service in a one-stop experience, by using cloud based tools, such as virtual containers [5].

\section{B. Mobile Learning and Mobile Service}

M-learning allows learners to participate in courses with mobile devices. Learners now have flexible options to access 'big' learning resources, whenever they want, wherever they are [7].

There were various drawbacks of mobile learning in the past decades, for example, learning resources for nonmobile devices cannot be directly adapted to mobile devices due to their indeterminacies of context, such as unpredictable network bandwidth, and specificities, such as different operation systems. Inspired by these, educational experts suggested that a key catalyst for m-learning to thrive is the services oriented and cloud computing paradigms, which harness economic benefits of large scale distributed systems, where computing resources are seamlessly integrated across geographical boundaries [8]. M-learning and mobile service are usually discussed together about their harmony because they have shared a lot of common features, such as location based specificity [9]. This convinces elearning practitioners that they can be facilitated mutually.

\section{Micro Learning}

Micro learning refers to short-term learning processes, which contains knowledge in small units. Typically, a micro learning activity is carried out through mobile devices within a time frame of 15 minutes [10]. As an emerging educational phenomenon, micro learning is more user-centric and requires different learning schedules than on-campus learning or even standard e-learning and m-learning [11]. Considering the massive acquirable OERs, how to set and select the right and appropriate objectives becomes a challenge for both OER providers and learners.

\section{OER SERVICE SELECTION, RECOMMENDATION AND COLD START PROBLEM}

\section{A. Micro Learning as a Service}

As stated in the Section I, a PLE over cloud is organized according to adaptive micro learning requirements, where MLaaS serves as a master service to search and find all available OERs and its associated services, and then it uses an online and an offline computing component to jointly make the decision of learning resource delivery. It aims to deliver learners adaptive micro learning resources in terms of their time availabilities by taking into account the specialties of the micro learning environment as well as the learners' 'highly-mobile' learning behaviors. MLaaS's framework has been well designed in terms of a genuine cloud standard, and its user model has been theoretically constructed by investigating comprehensive pedagogical literature and successful e-learning cases.

As the core of MLaaS, the Reasoning Engine consumes the results from all other services and transmits its output to the user interfaces straightforward. A knowledge base acts as the think tank of the system and works behind the Reasoning Engine. Basically, the knowledge base is responsible for semantic construction of the learner profile and OER representation. Every micro OER is measured in regards to its functional attributes and non-functional (QoS) attributes of the web service it belongs to [12][13].

However, a new problem appears. The system, MLaaS, has insufficient information about the learners, because both 'OER' and 'learner' are new to this emerging educational setting. This brings serious difficulties to launch the data processing work. The profile construction is impossible with insufficient information about the learner at the commencement of micro open learning.

\section{B. Research Challenges for Cold Start Problem for OER Service Delivery}

In computer science literature, widely used adaptive recommendation methods generally consist of two main categories, i.e., memory-based and model-based algorithms [14]. Although they have been found in many successful cases of recommender systems, for example, Amazon online store, it is usually difficult to provide reliable recommendations due to the insufficiency of initial data of ratings or preferences. This leads to the occurrence of cold start problem. Commonly the cold start problem is triggered by three factors: new community, new item and new users.

The cold start problem becomes more severe in the open learning, especially in micro learning through OERs [11]. Both open learning and OER are relatively new products, which are emerging in the very recent years. Meanwhile, the followers of this novel trend, no matter new education pursuers or regular learners migrated from other online learning modes, are forming a completely new community. 
On the other hand, the learning demands and expectations of learners engaged in open learning are much more practical than conventional university students. In other words, they are mostly self-regulated so that it is totally flexible for them to decide when to join or quit the online course at their own willingness, and switch among courses frequently [15]. Consequently, for OER providers, it is difficult to establish a model and update it accordingly for any individual learner because they do not have historical data in hand.

In micro open learning, or micro learning over OERs, it is very normal to find that learners take part in and deviate from the learning scenarios frequently, as well as turning on and off the learning activities at their own willingness. In other words, the overall situations of micro learning vary all the way, from individual to individual. Moreover, it is very common that freshmen join into open learning or existing learners unfold a brand-new course learning profile, at any time. All in all, there are a large number of new learners in open learning context; and new learners usually initiate access of new learning resources; and learners who consume learning resources in the same discipline will form as a new community.

If treated inappropriately, the cold start problem may lead to the loss of learners who previously engaged in open learning and then decide to stop using the OER delivery system or adopting the learning mode [16]. The reasons behind the situation are mainly due to the lack of accuracy in the recommendations received in that first stage, in which the learners have not yet cast a significant number of votes or ratings to feed the recommender systems. The scarcity of data affects the user satisfaction and then it can further affect the user acceptance of the new open learning mode.

Thus, if a learner is known scarcely by the OER system, the knowledge base should be able to treat it as a cold-start problem and tackle it by filling in the gaps with predicted data, in order to assist OER providers and instructors to guarantee the quality the first delivery, which can, furthermore, help promote the learner engagement in the ongoing open learning.

\section{AUGEMENTED ONTOLOGY CONSTRUCTION FOR MICRO OPEN LEARNING}

\section{A. Ontology Construction}

Naturally a workable knowledge base has a two-tier structure, a pattern level at the top and data level at the bottom [17]. For the pattern level, the ontologies are constructed based on conceptual graphs. By this means, the ontologies represent a formal way of the data processing workflow, and can drive the data processing with a priori knowledge and reduce the search space [18].

By accomplishing a comprehensive survey on literature in the fields of pedagogy, psychology, e-learning and mobile learning, we sorted out features that might play key roles in the micro open learning experience and achievement. These conceptual graphs also represent how features affected and interrelated with each other in the ongoing micro open learning process.

\section{1) Augmented Micro OER Ontology}

From the item-based view, we deepen the sights into the micro learning environment in particular and, for this reason, the general ontology of OER is augmented to adapt the needs of micro learning.

In the augmented micro OER ontology, an annotation of a micro OER is self-describing with metadata exploring its educational parameters, such as typology (video, audio, text, etc.), type of interaction (expositive, active, mixed, twoway), didactic model (e.g. inductive, deductive, learning by doing, etc.), and non-functional attributes, such as QoS, semantic density and so on [19]. Each node in the augmented OER ontology indicates a micro OER chunk. A chunk is the smallest unit in the micro learning settings, normally a finecut piece of an OER from its provider, and it has an apparently shorter time length (preferably less than 15 mins) than its original shape. It can be a mini concept or knowledge point, tinier than what the teachers used to deliver; or it can be a cut of course video or lecture notes, or a course settings come along with a concept, such as assessment, task, reading material and so on [20].

There is no completely independent chunk and each of them is part of a relational web rather than merely a conceptual object [19]. This ontology is used to explicitly classify the OERs to recommend among a pedagogically defined set of distinctive main concepts, fed as the raw material in the reasoning process of MLaaS [16] [21].

\section{2) Augmented Micro Learning Learner Profile} Taxonomy

From the user-based view, the main ontology, on which all learner profiles are based, is named as the Benchmark ontology, where the element Learner is put in the center of the graph [14]. As the instances of the presetting domain ontologies, a specific learner profile oriented to micro learning is a set of nodes from the Benchmark ontology versus a node in the augmented micro OER ontology. It contains plenty of annotations in terms of their learning behaviors and context.

\section{B. OER Investigation}

For the bottom level (i.e. data level) of the knowledge base, to technically operate the semantic learner profile and knowledge base construction for micro open learning, data filled in the graphs come from two sides, the explicit data collection (e.g. through mandatory requests) and implicit data tracking (e.g. automatic extraction) [21].

In addition, rather than developing the domain ontology for OERs by ourselves, a general structure of courseware ontology is built jointly by making use of existing ontologies, which has been extracted from main OER providers, such as universities involved in major open courseware alliances (e.g. participating institutions in edX $\mathrm{X}^{1}$ ), or from the Linked Open Data Cloud community ${ }^{2}$ [22]. The investigation over 'big' open learning data comes up to the OER side. Among the massive OERs, three types of relations are mainly targeted to be foreseen:

\footnotetext{
${ }^{1}$ https://www.edx.org/schools-partners

2 http://lod-cloud.net/
} 
- ConsistsOf is an inclusion relation. This relation can be generally found between two OERs or one OER and one micro OER. Two items with this relation are located in different hierarchies of the augmented micro OER ontology.

- $\quad$ RequiredSequence is a strong order between two items (OER or micro OER), where the former micro OER is necessarily to be learnt before the latter one, due to course setting and educational consideration.

- RecommendedSequence is a weak order relation between two items (OER and micro OER), where the former micro OER is suggestively to be learnt before the latter one, according to the instructors' guidance, but it is not mandatory.

- $\quad$ Certainly, two items (OER or micro OER) can have no relation at all.

- Both relations regarding sequence can be inherited by entities' descendants, for example, if there is a RecommendedSequence $\left(R_{1}, R_{2}\right)$ indicating an OER $R_{l}$ is preferably learnt prior to $R_{2}$, then, for $M R_{l} \in R_{l}$ and $M R_{2} \in R_{2}$, there is a RecommendedSequence ( $\mathrm{M} R_{l}$, $\left.\mathrm{M} R_{2}\right)$

\section{ONTOLOGICAL APPROACH FOR COLD-START PROBLEM}

\section{A. Representation of Learner Profile}

Adopting ontologies as the basis of the learner profile is crucial in addressing the cold start problem in micro OER delivery. It allows the initial learner behavior to be matched with existing and pre-known knowledge in the ontologies and relationships among them.

The learner profile is managed by MLaaS by two parts: the static part and the dynamic part. The static part can be represented by a vector, which contains the demographic and educational information. By matching these two augmented ontologies, respectively for item and user, the dynamic part of a learner node is denoted as a pair, $L_{j}=\left\{M R_{u}, M L_{j}\right\}, L_{j} \in L$. Herein, the element $M R_{u}$ denotes the $u^{\text {th }}$ micro OER, as introduced in the Subsection A of the Section IV, which is a particular version of the micro OER ontology, and a three dimensional element $M L_{j}\left\{P_{u, j}, T A_{j}, D_{j}\right\}$ is exclusive to $j^{\text {th }}$ learner during the micro learning process. Herein, the element $P_{u, j}$ indicates the learner's preference, $T A_{j}$ indicates the $j^{\text {th }}$ learner's instantly time availability, and $D_{j}$ denotes the level of distraction in terms of the given learning environment and surroundings.

Whenever MLaaS gathers any information from the learner's learning process over OER, the learner profile will be updated in regards to $M L_{j}$.

\section{B. Preference Propagation}

With the cold start condition for the first micro OER delivery, a learner is required to quickly mark down a preference on a specific micro OER. Consequently, a spreading activation approach is applied to maintain the preference against its parent node (i.e., the $R_{v}$ is the $v^{\text {th }}$ OER where the $M R_{u}$ derived from) as well as updating learner profile. It propagates the learners' preference upwards the hierarchy of micro OER ontology based on activation values. In other words, the preference has been obtained from a micro OER to its ancestor and spread in its superclass (i.e., OER) level. An example of the spreading activation is shown in Figure 1.

A partial view of augmented micro OER ontology in 'information technologies' area is shown in Figure 4. Particularly, it describes an 'e-business' OER from an Australian provider, OpenLearning ${ }^{3}$. At the bottom level of the ontology, the nodes depicted with oval shape typically conform to the standard of micro OER. The red integers shown in nodes with rectangle shape are preference values from a learner versus target OERs. The algorithm 1 is proposed to execute the process of preference propagation.

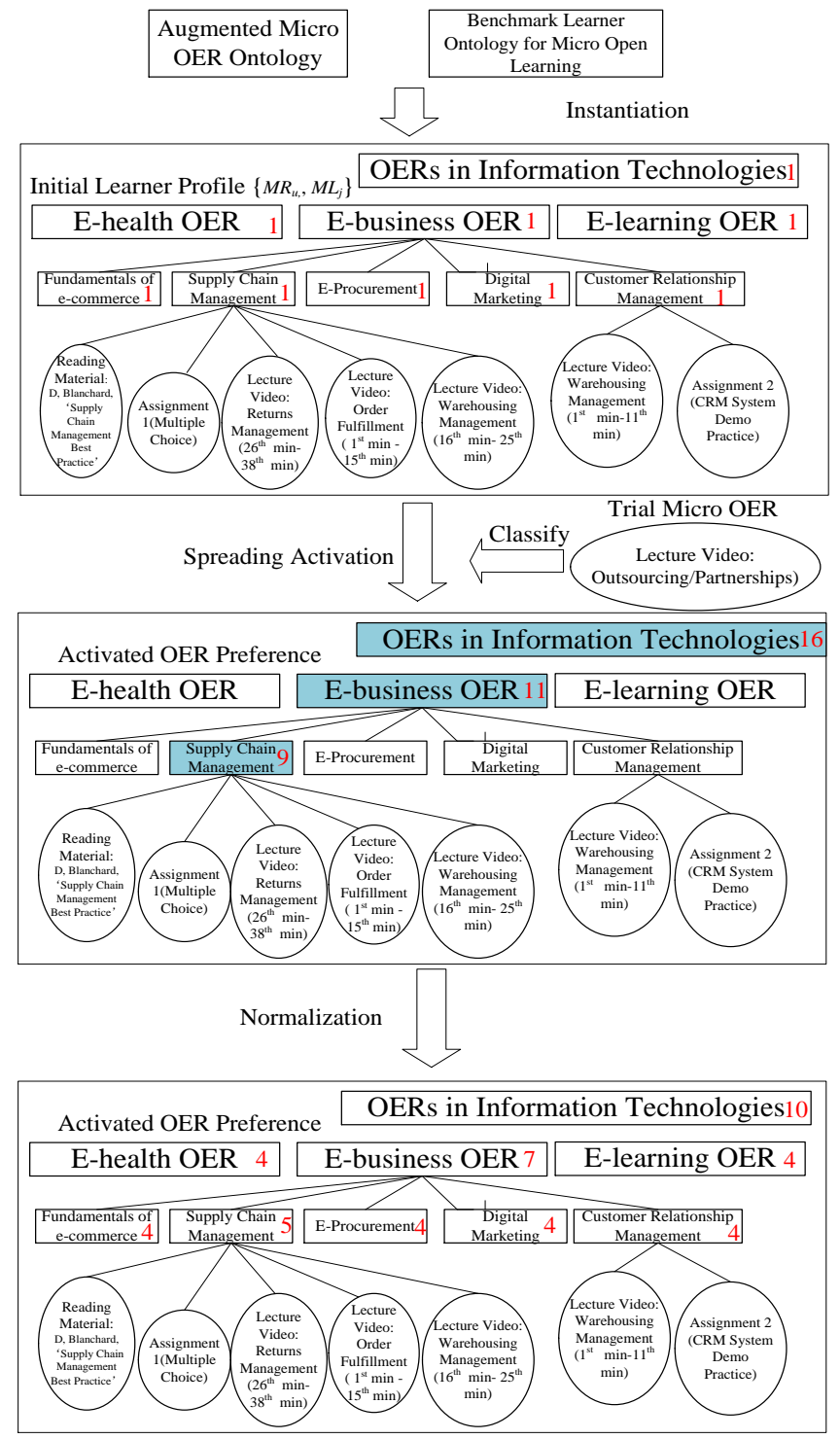

Figure1. Partial View of the Augmented Micro OER Ontology and Spreading Activation for a Learner's Preference on OER

\footnotetext{
${ }^{3}$ https://www.openlearning.com/
} 


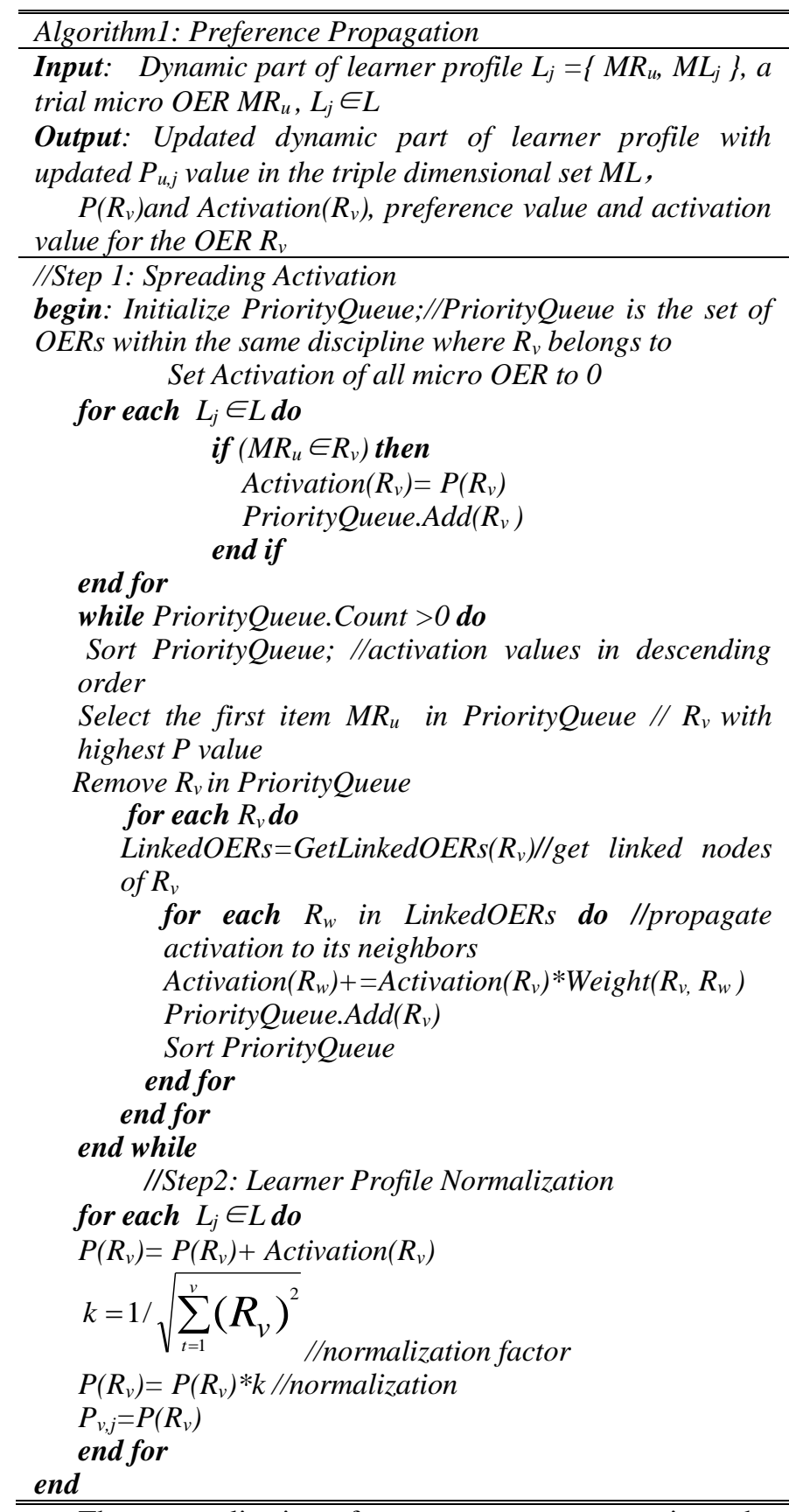

The normalization factor acts on preventing the propagated preferences from escalating continuously to such an extent that exceeds a reasonable range, which could result in difficulty of data processing in the forthcoming process. The confidence degree for the propagated preference of OER is recorded as $C D\left(P_{v, j}\right)$.

\section{Instant Time Availability}

The system is able to obtain explicit information on how long the learner can (or would like) to spend on a micro OER through mobile devices in the real time. As a mandatory request, a learner is required to input his or her instant time availability at the beginning of every micro learning activity.
According to the system setting, suggestively the instant time availability, $T A_{j}$, is represented by an integer from 1 to 15. However, if the learner is not sure how long he is able to spend on the micro OER at once, he is free to leave a time span, which can be continuous integers in the same range.

\section{Learner Feature Prediction}

\section{1) Demographic Classification}

In [18], we have discussed the key issues that might cause distraction in micro open learning, which generally came from two sides, the social side and environmental side.

In addition, MLaaS investigates existing learners' degree of distraction as reference, and senses every learner's location information through built-in functions in mobile devices. Based on the given taxonomy and augmented ontology, we carry out a demographic classification that aims to cluster learners into cohorts, in order to match them with micro-pieces of OERs.

The mechanism of classification is designed as, learners who have similar static information, involving employment and/or education background, occupation, and similar learning environment/location, are more likely to face similar level of distraction. For the same reason, their overall time availabilities would more likely fall in the same range. Herein MLaaS tries to associate a learner into a pre-clustered learner group, by applying the stereotyping technique to fulfill the requirement of demographic classification.

For a newly joined learner, $L_{j}$, an ensemble method of a binary classifier and a one-against-all model is utilized to obtain multi-class classification achievements [23][24], in order to predict its category, $C_{j}$. The system is trained with existing set of learners, $L$. Typical binary classification techniques, i.e., C4.5 decision tree [25][26] or Naive Bayes classifier [27] can be employed to serve as the base algorithm (i.e. training algorithm $F$ in Algorithm 2) in order to produce a suitable classifier, $C F_{k}$. A new learner $L_{j}$ is classified with the label $k$, whose $C F_{k}$ produces the highest value of $\hat{y}$.

The algorithm 2 shows the process of classifier selection. Algorithm2: New Learner Classification (one-against-all )

Input: Sample (Current learner set L), Labels y ( where $y_{i}$ is the label for a sample learner $L_{i}$ and $\left.y_{i} \in\{1,2, \ldots K\}\right)$,

Training Algorithm $F$, a new learner $L_{j}$ Output: category of the new learner $L_{j}, C_{j}$

begin:

for each $k$ in $\{1,2, \ldots K\}$ do set a new label vector $z_{i}$ for $y_{i}$, if $\left(y_{i}=k\right)$ then

$z_{i}=1$

else

$z_{i}=0$

end if

$C F_{k}=F(L, z) \quad / /$ use binary classification technique to produce classifiers

end for

for $L_{j} \in L d o$ 


$$
\hat{\mathrm{y}}=\arg \max _{k \in\{1,2 \ldots \mathrm{k}\}} C F_{k}\left(L_{j}\right)
$$

\section{end for}

output $C_{j}=k / /$ category of the new learner $L_{j}$ end

Given $L_{j}$ is categorized into the $C_{k}$, afterwards, the learner's neighborhood, $N B_{j}$, is calculated by the Algorithm 3. This aims to match a new learner's category with an existing learner's category.

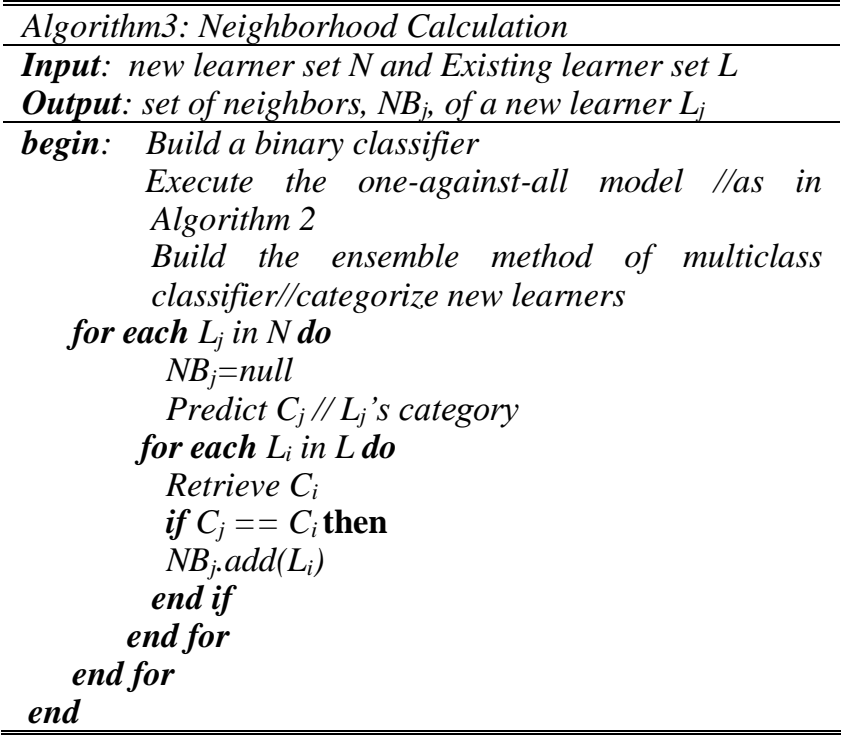

Hence, the demographic classification is realized according to learners' static and location information. Once new learners join into the open learning scenario, MLaaS responds immediately to classify them into clusters.

2) Similarity Measure between Two Learners

MLaaS is responsible to find the similar existing learners in the discovered demographic categories, so as to recommend them micro OERs that were recognized as suitable to learn in a given time span, situation and environment.

Learners' learning location information is sensed from the location service embedded in the mobile devices. Thus, the similarity between two learners, $L_{i}$ and $L_{j}$, is evaluated using the equation (1).

$$
\operatorname{sim}(i, j)=\left[\left(\sum_{l=1}^{m} S_{l} W_{l}\right)^{2}+\left(S L \mathbf{o}_{i, j} W_{i, j}\right)^{2}\right]^{1 / 2}
$$

where $S_{l}$ is the similarity value of the $l^{\text {th }}$ attribute in the static part of learner profile and the $W_{l}$ is its corresponding weight. $S L o_{i, j}$ denotes their similarity on location and $W_{i, j}$ denotes the weight for location factor.

\section{3) Distraction Prediction}

Thus, in terms of the equation (2), the distraction value can be estimated in accordance with the action that any member in a same cluster indicates the predicted distraction level.

$$
D_{j, L D_{a}}=\frac{1}{2} *\left(\frac{\sum \operatorname{sim}(i . j) \bullet d_{i, L_{a}}}{\sum \operatorname{sim}(i, j)}+d_{j, L_{a}}\right)
$$

where $d_{j, L o a}$ is the self-identified degree of distraction the learner $L_{j}$ felt in the location $L o_{a}$, acquired by mandatory request. This follows the expectation that the learners who have similar general situation (i.e. social factors) and surroundings (i.e. environmental factors) are in high probability to have similar degree of distraction.

The confidence degree for the predicted distraction is depicted as $C D\left(D_{i, L o a}\right)$.

\section{E. Integration of Recommendation Results}

\section{1) Downwards Propagation}

In the Subsection A we have merely obtained the preference of a learner on an 'entire' OER rather than on a micro OER, now the preference values are again propagated downwards the ontology hierarchy. Consequently, each micro OER node receives an estimated preference value from its ancestor. This propagation process is executed with a decay factor. For each micro OER the final preference value, $P_{u, j}$, can be calculated use the following equation (3).

$$
P_{u, j}=\frac{\sum P_{R, j} C D\left(P_{R, j}\right)+\sum P_{v, j} C D\left(P_{v, j}\right) Q(u, v)}{\sum C D\left(P_{R, j}\right)+\sum C D\left(P_{v, j}\right) Q(u, v)}
$$

where $R$ is the set of all the nodes in the higher hierarchy than $M R_{u}, R_{v}$ is a direct ancestor of node $M R_{u}$ and $Q(u, v)$ depends on the count of level between $M R_{u}$ and $R_{v}$.

$$
Q(u, v)=L(|u v|)=\left\{\begin{array}{l}
\mathrm{L}(0)=1 \\
L(l)=(1-\lambda) L(l-1)
\end{array}\right.
$$

and the confidence degree for the descendant node, in regards to the $P_{u, j}$, is calculated as the average of the confidence values in its ancestors, decreased by a decay factor, $\mu$.

$$
C D\left(P_{u, j}\right)=\frac{\sum C D(R)}{|\mathrm{R}|}-\mu
$$

As far as all values of the three attributes, denoting preferences, instant time availability and degree of distraction, in the set $M L$ are settled, a complete learner profile is constructed from the initial little-known information by the MLaaS.

2) Micro OER Screening and Rules

For each micro OER, once MLaaS has acquired its final preference value and confidence degree, those nodes, which do not meet the minimum requirement of confidence degree, is rejected by the system.

To generate a list of recommended micro OERs, where the ones with higher learners' interests are placed at the top. For two micro OERs $M R_{u}$ and $M R_{w}$, their sequence is determined according to some heuristic rules which are defined in accordance with the extraction of three kinds of relations discussed in the Subsection B of the Section IV. These rules are executed sequentially with priority.

1. If there is a RequiredSequence relation between these two micro OERs, the prerequisite one is placed above (refer to the Subsection B of the Section IV).

2 . If the preference regarding these two OERs, $P_{u, j}$, $P_{w, j}$, the former one is higher than the latter one, then the $M R_{u}$ is above $M R_{w}$ 
3. If, in the absolute terms, the confidence degree $C D\left(P_{u}, j\right)$ is high and the $C D\left(P_{w, j}\right)$ is low, then the $M R_{u}$ is above $M R_{w}$.

4. If there is a RecommendedSequence relation between these two micro OERs, the one which is suggested to be accessed first is placed above (refer to the Subsection $\mathrm{B}$ of the Section IV).

5. The micro OER, which is more related to the learners' education background, or falls in the relevant disciplines or inter-disciplines is placed with priority if the disciplinary difference between these two candidate micro OERs is obvious.

6. Otherwise the recommended micro OER list is randomly ordered if none of the above rules applies.

Herein, the first rule is deemed as a hard rule which should be strictly obeyed and the rest rules are soft rules which can be violated with educational consideration, from case to case.

\section{3) Recommendation Results Optimization}

MLaaS consumes the value $P$ and $D$ in conjunction with their $T A$ to compare with the attributions and requirements annotated in the metadata of the augmented OER ontology.

The next step is to integrate the outcomes from the Subsection C of Section V, a fitness function will convert these selected multidimensional arrays into one variable. Hence, this problem is hereby properly transferred to a multiobjective optimization problem.

To initiate the constrained multi-objective optimization, candidate learning path solutions (chromosomes) are randomly generated where each of them is a learning path with a series of micro OERs. For a chromosome, its violation degree is investigated by examining the relations between each contiguously prior/posterior micro OER pair against the first 5 rules listed in the previous subsection, and then summing up. For such pair in a chromosome, its violation degree, $V D\left(M R^{t}, M R^{t+1}\right)$, is calculated by the weighted sum of its violations against rule 2 to rule 5 , respectively, where $M R^{t}$ is the $t^{\text {th }}$ micro OER in $k$ and $M R^{t+1}$ is the $t+l^{t h}$. The higher the violation degree is, the more serious the candidate learning path violates the rules. The violation degree of a candidate learning path, $k$, is calculated using the following equation (6)

$$
V D_{k}=\sum V D\left(M R^{\mathrm{t}}, M R^{t+1}\right)
$$

Thereafter, let the variable $R A_{u}$ denote the degree of required attention of a given micro learning resource, $M R_{u}$, whose real-time suitability for micro learning, $R T_{u, j}$, is calculated by comparing with the learner, $L_{j}^{\prime}$ 's predicted distraction, using the following equation (7):

$$
R T_{u, j}=\left\{\left(R A_{u}\right)^{2}+\left[C D\left(D_{j, L_{a}}\right) * D_{j, L_{a}}\right]^{2}\right\}^{1 / 2}
$$

Hence, for the candidate learning path, $k, R T_{k, j}$ denotes the sum of the real-time suitability of micro OERs it contains. Similarly, $P_{k, j}$ sums up all the predicted preferences from the learner $L_{j}$ versus micro OERs that $k$ contains.

$$
\eta=\min \left(\alpha V D_{k}+\beta R T_{k, j}+\gamma / P_{k, j}^{1}+\delta / P_{k, j}\right)
$$

where $a, \beta, \gamma$ and $\delta$ serve as weight for each variable and suggestively $a>\beta>\gamma>\delta, P_{k, j}^{l}$ denotes the $L_{k}$ 's preference value of the first micro OER in the candidate learning path $k$.

The algorithm 4 indicates typical steps of make the first recommendation

Algorithm4: Micro OER Recommendation in a Cold Start Condition

Input: $P_{u, j}$ (the Learner $L_{j}$ 's predicted reference to the micro OER $\left.M R_{u}\right), D_{j, \text { Loa }}$ (predicted distraction level), $C D\left(P_{u, j}\right)$ and $C D\left(D_{i, L o a}\right)$ (their confidence degree), $R A_{u}$ (the degree of required attention of $M R_{u}$ ), $T A_{j}$ (the instant time availability ), rules $\left(1^{\text {st }}-6^{\text {th }}\right)$

Output: the tag of a micro OER which acts as the first delivery

begin: Randomly generate candidate learning paths as chromosomes

for each chromosome $k$ do Select micro OER it contains

for each $M R_{u}$ in a chromosome $k$, Calculate its $P_{u, j}$ and $C D\left(P_{u, j}\right)$. Import $D_{j, \text { Loa }}, C D\left(D_{i, L o a}\right)$ and $R A_{u}$ Calculate its $R T_{u, j}$ end for Calculate $k$ 's $V D_{k}$ Use equation (8) to evaluate its fitness $\eta$

end for

while iteration times $<$ max iteration time do

apply heuristic approach to generate new candidate solutions

for each new chromosome $k^{\prime}$ do

check time length of the first micro OER in $k^{\prime}, T L_{k^{\prime}}^{1}$

if $T L_{k}^{l}$, is in the range of $T A_{j}$ keep $k$ '

otherwise

reject $k$ '

end if

evaluate the fitness of $k$ ', $\eta$, using equation (8)

end for

replace chromosomes with higher $\eta$

end while

output the selected chromosome $k$ " with minimum $\eta$ and satisfied $T L_{k}^{l}$,

select the first micro OER in $k$ "' as the first delivery end

By this means, the heuristic algorithm 4 infers a suitable micro OER as the first attempt of learning resource recommendation for a learner at the commencement of the novel micro open learning experience via MLaaS.

Along with the successful launch of solution to the wellknown cold start problem in micro learning, learners' upcoming behaviors will be continuously acquired by MLaaS to feed the Reasoning Engine.

\section{CONCLUSION}

A software as a service, MLaaS, was designed to deliver adaptive micro OERs, which works as a master service to 
search and select all available OERs and their affiliated services online. The Reasoning Engine in MLaaS consumes users' personal information, instant time availabilities, specificities of mobile environment and micro OERs' features to make decision intelligently. However, since both the system and users are new, the lack of learner information in MLaaS brings difficulties to the commencement of adaptive micro open learning as well as MLaaS operation.

For this reason, in this paper we introduce a service based approach to deal with the cold start problem in the recommendation of micro OERs over a preferably cloud platform. We primarily focus on the knowledge base construction against the sparsity of data. Augmented semantic profiles of learners and OERs are built previously to model the structure and features of their information. Sequentially, a detailed approach is provided to deal with the cold start problem by predicting learners' features from the initial little-known information.

Our future work will involve the evaluation of the proposed approach by measuring the prediction accuracy and engaging real learners to compare the quality of recommendations.

\section{REFERENCES}

[1] J. Hylen, D.V. Damme, F. Mulder and S. D' Antoni, 'Open Educational Resources: Analysis of Responses to the OECD Country Questionnare', OECD Education Working Papers No. 76, June 2012.

[2] J. Hilton, 'Open Educational Resources and College Textbook Choices: a Review of Research on Efficacy and Perceptions', Educational Technology Research and Development, vol.64, no. 4, pp.573-590, 2016.

[3] J. Baggaley, 'MOOC Rampant', Distance Education, vol. 34, no. 3 pp. 368-378, 2013.

[4] G. Sun, T. Cui, S. Chen, W. Guo and J. Shen, 'MLaaS: A Cloud Based System for Mobile Micro Learning in MOOC', 4th IEEE International Conference on Mobile Service (MS), New York, US, July 2015, pp.120-127.

[5] A. Corbi and D. Burgos, 'OERaaS: Open Educational Resources as a Service with the Help of Virtual Containers', IEEE Latin America Transactions, vol.14, no. 6, pp. 1-7, 2016.

[6] R. Hernandez, C. Gutl, H.R. Amado-Salvatierra, 'Cloud Learning Activities Orchestration for MOOC Environments', the $3^{\text {rd }}$ International Workshop on Learning Technology for Education in Cloud, Santiago, Chile, Sep 2014, pp. 25-36.

[7] M. Wang, Y. Chen and M. J. Khan, 'Mobile Cloud Learning for Higher Education: A Case Study of Moodle in the Cloud', The International Review of Research in Open and Distributed Learning, vol. 15 , no. 2, pp. 255-267, 2014.

[8] M. Sarrab, N. Alalwan, O. Alfarraj and A. Alzahrani, 'An Empirical Study on Cloud Computing Requirements for Better Mobile Learning Services', International Journal of Mobile Learning and Organisation, vol. 9, no. 1,2015 .

[9] H.T.Dinh, C. Lee, D. Niyato and P. Wang, 'A Survey of Mobile Cloud Computing: Architeture, Applications and Approaches', Wireless Communications and Mobile Computing, vol. 13, no. 18, pp. 1587-1611, 2011.

[10] D. Kovachev, Y. Cao, R. Klamma and M. Jarke, 'Learn-as-you-go, New Ways of Cloud Based Micro-learning for the Mobile Web', 10th International Conference on Web-based Learning, Hongkong, December 2011.
[11] P.A.Bruck, L.Motiwalla and F. Foerster, 'Mobile Learning with Micro-content: A Framework and Evaluation', 25th Bled eConference, Bled, Slovenia, 2012, pp.527-542.

[12] G. Sun, T. Cui, W. Guo, G. Beydoun, D. Xu and J. Shen, 'Micro Learning Adaptation in MOOC: a Software as a Service and a Personalized Learner Model', the 14th International Conference on Web-based Learning (ICWL), Guangzhou, China, November 2015, pp.174-184.

[13] L. Wang and J. Shen, 'Multi-phase ant colony system for multi-party data-intensive service provision', IEEE Transactions on Service Computing, vol. 9, no. 2, pp. 264-276, 2016.

[14] A. Sieg, B. Mobasher and R. Burke, 'Ontology-Based Collaborative Recommendation', the 8th Workshop on Intelligent Techniques for Web Personalization and Recommender Systems, Hawaii, USA, June 2010, pp. 20-32.

[15] S. Miranda, G. R. Mangione, F. Orciuoli, M. Gaeta and V. Loia, 'Automatic Generation of Assessment Objects and Remedial Works for MOOCs', 12th International Conference on Information Technology Based Higher Education and Training, Antalya, Turkey, Octorber 2013.

[16] G. Sun, T. Cui, G. Beydoun, S. Chen and J. Shen, 'Profiling and Supporting Adaptive Micro Learning on Open Education Resources' the $4^{\text {th }}$ International Conference on Advanced Cloud and Big Data (CBD), Chengdu, China, August 2016, pp.158-163.

[17] E. Cambria, D. Olsher and D. Rajagopal, 'SenticNet3: a Common and Common-sense Knowledge Base for Cognition-driven Sentiment Analysis', the $28^{\text {th }}$ AAAI Conference on Artificial Intelligence (AAAI), Quebec, Canada, July 2014, pp.1515-1521.

[18] D. Dou, H. Wang and H. Liu, 'Semantic Data Mining: a Survey of Ontology-based Approaches', the $9^{\text {th }}$ IEEE International Conference on Semantic Computing (ICSC), California, USA, February 2015, pp. 244-251.

[19] S. Miranda and G. Albano, 'Personalized Learning in Mathematics', Journal of E-learning and Knowledge Scociety, vol. 11, no.1, pp.25$42,2015$.

[20] G. Sun, T. Cui, J. Yong, J. Shen and S. Chen , 'MLaaS: a Cloudbased System for Delivering Adaptive Micro Learning in Mobile MOOC Learning'. IEEE Transactions on Services Computing, online first http://dx.doi.org/10.1109/TSC.2015.2473854

[21] A. Moreno, A. Valls, D. Isern, L. Marin and J. Borras, 'SigTur/EDestination: Ontology-Based personalized Recommendation of Tourism and Leisure Activities', Engineering Applications of Artificial Intelligence, vol.26, no.1, pp.633-651, 2013.

[22] N. Capuano, L. Dell'Angelo, F. Orciuoli, 'Ontology Extraction from Existing Education al Content to Improve Personalized e-Learning Experiences', 3rd IEEE International Conference on Semantic Computing, Berkeley, USA, September 2009, pp. 577-582.

[23] J. Milgram, M. Cheriet and M. Sabourin, 'One against One or One against all: Which One is Better for Handwriting Recognition with SVMs', 10th International Workshop on Frontiers in Handwriting Recognition, France, October 2006.

[24] M. Galar, A. Fernandez, E. Barrenechea, H. Bustince and F. Herrera, 'An Overview of Ensemble Methods for Binary Classifiers in Multiclass Problems: Emperimental Study on One-vs-One and One-vs-All Schemes, Pattern Recognition, vol. 44, pp.1761-1766, 2011.

[25] S.B.Kotsiantis, 'Supervised Machine Learning: a Review of Classification Techiniques', Informatica, vol.31, no. 3, pp. 249-268, 2007.

[26] K. Polat and S.Gunes, 'a Novel Hybrid Intelligent Method Based on C4.5 Decision Tree Classifier and One-against-All Approach for Multi-class Classification Problems', Expert System with Applications, vol.36, pp.1587-1592, 2009.

[27] L. A. Dalton and E. R. Dougherty, 'Optimal Classifiers with Minimum Expected Error within a Bayesian Framework-Part II: Properties and Performance Analysis', Pattern Recognition, vol. 46, no. 5, pp. 1288-1300, 2013. 